\title{
ON THE RESULTANT OF A SYSTEM OF FORMS HOMOGENEOUS IN EACH OF SEVERAL SETS OF VARIABLES*
}

\author{
BY
}

NEAL H. McCOY

INTRODUCTION

One of the most fundamental problems in the theory of elimination may be stated as follows. Let

$$
f_{i}^{\prime} \quad(i=1,2, \cdots, n)
$$

be a set of $n$ general forms homogeneous in the $n$ variables $x_{1}, x_{2}, \cdots, x_{n}$; to determine the polynomial in the coefficients of these forms whose vanishing is a necessary and sufficient condition that the forms (1) simultaneously vanish for a set of values, not all zero, of the variables $x_{1}, x_{2}, \cdots, x_{n}$. This polynomial is called the resultant of the system of forms (1). From this standpoint a numerical factor in the resultant is of no consequence though in certain cases it is desirable to introduce some convention as to such a factor.

The most important properties of the resultant of the system (1) are well known and have been obtained by various authors in a variety of ways. $\dagger$ We give a brief account of the method used by König $\ddagger$ as it is of particular importance in the sequel.

Let us denote by

$$
f_{i} \quad(i=1,2, \cdots, n)
$$

the general non-homogeneous polynomials obtained from (1) by placing one variable, say $x_{n}$, equal to unity in each form. We now consider the module defined by these polynomials, that is, the system of all polynomials of the form

$$
\phi_{1} f_{1}+\phi_{2} f_{2}+\cdots+\phi_{n} f_{n}
$$

* Presented to the Society, September 9, 1931; received by the editors May 21, 1932. This paper was practically completed while the author was a National Research Fellow at Princeton University.

† See the Encyklopädie, vol. 1, pp. 260-273; also J. König, Einleitung in die allgemeine Theorie der algebraischen Grössen, Leipzig, Teubner, 1903, chapter VI; F. S. Macaulay, Algebraic Theory of Modular Systems, Cambridge University Tracts, No. 19, 1916, pp. 3-17; O. Perron, Algebra I: Die Grundlagen, Göschens Lehrbücherei, vol. 8, 1927.

¥ Op. cit. The exposition given by König is based on earlier work of F. Mertens. For references see König, op. cit., p. 271. 
where the $\phi_{i}$ are also polynomials in $x_{1}, x_{2}, \cdots, x_{n-1}$. It may be shown that there exists one and only one polynomial $R$ in the coefficients of the polynomials $\left(1^{\prime}\right)$ satisfying the following two conditions: (i) $R$ is a member of the module (2), and (ii) $R$ is an irreducible function of the coefficients of these general polynomials. This polynomıal $R$ is defined by König to be the resultant of the polynomials $\left(1^{\prime}\right)$ and also of the forms (1). The resultant as thus defined is identical with the polynomial in the coefficients whose vanishing is a necessary and sufficient condition that the forms (1) vanish for a common set of values of the variables. However, this fact is not the center of interest from this point of view. The usual properties of the resultant may be obtained by a method of induction.

It is the purpose of the present paper to consider a certain generalization of the concept of resultant from the point of view of modular systems. Let

$$
F_{i}{ }^{\prime} \quad\left(i=1,2, \cdots, 1+\sum_{j=1}^{r} \alpha_{j}\right)
$$

denote a set of general forms homogeneous in the variables of each of $r(\geqq 1)$ sets, there being $\alpha_{j}+1$ variables in the $j$ th set $(j=1,2, \cdots, r)$ and each $\alpha_{j} \geqq 1$. Sylvester* seems to have been the first to consider the concept of a resultant of forms of the type (3) and although he did not define the resultant of such a set of forms, he stated without proof a general theorem regarding the degree and weight of the resultant. This theorem is essentially our Theorem 3 (c,d) below.

A definition and a brief discussion of the resultant of the system (3) was given by Lasker $\uparrow$ from a point of view somewhat similar to that of the present paper. However, Lasker was not primarily interested in the structure of the resultant but in its use in generalizing certain theorems in the theory of modules and ideals.

Certain special cases have been studied by different authors with, of course, varying points of view. Sylvester and Muir $\ddagger$ have discussed the resultant of a system of forms linear in each of two sets of variables and have expressed the resultant in the form of a determinant in two or three different

* J. J. Sylvester, On the degree and weight of the resultant of a multipartite system of equations, Proceedings of the Royal Society of London, vol. 12 (1862-63), pp. 674-76, or Mathematical Papers, vol. 2, pp. 329-330.

† E. Lasker; Zur Theorie der Moduln und Ideale, Mathematische Annalen, vol. 60 (1905), pp. 105-107.

$\ddagger \mathrm{T}$. Muir, The resultant of a set of homogeneous lineo-linear equations, Transactions of the Royal Society of South Africa, vol. 2 (1910-12), pp. 373-380; J. J. Sylvester, On a question of compound arrangement, Proceedings of the Royal Society of London, vol. 12 (1862-63), pp. 561-563, or Mathematical Papers, vol. 2, pp. 325-326. 
ways. The case of three double binary forms has been considered by Moore and by the present author with the object of expressing the resultant in determinantal form.*

In Part I we give a definition of the resultant of a system of forms of type (3) and deduce some of its fundamental properties. The outline of procedure is essentially that of König $\dagger$ for the classical case $r=1$. Some of his results can be carried over immediately to this more general case and with one or two exceptions we shall refer to König for the proofs wherever possible. However we give in some detail the demonstrations that involve any essential modification or extension.

Part II consists of a generalization of Sylvester's dialytic method of elimination to certain cases of forms of the type here considered. The main result is Theorem 4 . As special cases of this theorem we obtain the resultant in the form of a determinant for (i) two ordinary binary forms of arbitrary degrees (Sylvester's determinant); (ii) multiple binary forms of arbitrary degrees in the variables of one set, all the forms being of the same degree in the variables of any other given set; and (iii) forms linear in any number of sets of variables, there being an arbitrary number of variables in each set. The form of the determinant in the third case for two sets of variables is different from the determinants obtained by Muir to which reference was made above.

In general, we obtain more than one determinantal expression for the resultant as the form of the determinant occurring in the statement of Theorem 4 depends in a certain way upon the notation adopted.

\section{Definition and fundamental properties of the Resultant}

1. Notation and preliminary remarks. Let us denote by $x_{j 1}, x_{j 2}, \cdots$, $x_{i, \alpha_{j+1}}$ the variables of the $j$ th set occurring in the forms $(3)(j=1,2, \cdots, r)$. We shall henceforth let $m$ denote the quantity $1+\sum_{j=1}^{r} \alpha_{j}$. The degree of $F_{i}^{\prime}$ in the variables of the $j$ th set will be indicated by $n_{i j}(i=1,2, \cdots, m$; $=1,2, \cdots, r)$. We assume throughout that each $n_{i j}>0$; that is, each of the sets of variables actually appears in each form. $\ddagger$

* T. W. Moore, Extended results in elimination, Annals of Mathematics, vol. 30 (1928), pp. 92100 ; N. H. McCoy, On the resultant of three double binary forms, Ibid., vol. 33 (1932), pp. 177-183. We include here the following additional references which have some relation to the subject of this paper: A. Brill, Ueber Elimination aus einem gewissen System von Gleichungen, Mathematische Annalen, vol. 5 (1872), pp. 378-396; T. Muir, Elimination in the case of equality of fractions whose numerators and denominators are linear functions of the variables, Transactions of the Royal Society of Edinburgh, vol. 45 (1906), pp. 1-7; K. Th. Vahlen, Ueber den Grad der Eliminationsresultante eines Gleichungssystems, Journal für die Reine und Angewandte Mathematik, vol. 113 (1894), pp. 348-352.

$\dagger$ Op. cit. It will be understood henceforth that any reference to this author refers to this book.

$\ddagger$ A resultant exists under certain conditions even if this restriction is not made. Cf. Lasker, loc. cit., p. 106. 
It will be convenient at present to consider in place of the homogeneous forms (3) the general non-homogeneous polynomials

$$
F_{i} \quad(i=1,2, \cdots, m)
$$

obtained from them by placing $x_{j, \alpha_{j}+1}=1(j=1,2, \cdots, r)$ in each form. By a general polynomial we shall mean henceforth a polynomial obtainable in this way from a general form of the type (3).

The totality of variables in all the various sets may be denoted by $x$, and $a$ will indicate the aggregate of coefficients in all the forms under discussion. Thus $\phi(a, x)$ will represent a polynomial in the coefficients of the set (4) and in certain of the variables.

Let $\gamma_{i}$ denote the constant term in $F_{i}$, that is, the term containing none of the variables. By $[\phi(a, x)]$ we shall indicate the polynomial obtained from $\phi(a, x)$ by substituting $\gamma_{i}-F_{i}$ for $\gamma_{i}(i=1,2, \cdots, m){ }^{*}$ If we make this substitution only on $\gamma_{i}(i=2,3, \cdots, m)$, we shall indicate the resulting polynomial by $[\phi(a, x)]_{1}$. It is seen that

$$
\phi(a, x) \equiv[\phi(a, x)]_{1}+H_{2} F_{2}+\cdots+H_{m} F_{m},
$$

where the $H$ 's are polynomials. This may be expressed in the usual notation,

$$
\phi(a, x) \equiv[\phi(a, x)]_{1} \quad\left(\bmod F_{2}, F_{3}, \cdots, F_{m}\right) .
$$

For the sake of completeness we now prove two theorems which are of fundamental importance. The proofs do not differ in any essential from the corresponding proofs in the special case $r=1$ but the second in particular illustrates a method of proof which is important in establishing later theorems.

THEOREM $1 . \dagger$ If

$$
\phi(a, x) \equiv 0 \quad\left(\bmod F_{1}, F_{2}, \cdots, F_{k}\right),
$$

then $\phi$ actually contains all the coefficients occurring in $F_{1}$, or

$$
\phi(a, x) \equiv 0 \quad\left(\bmod F_{2}, F_{3}, \cdots, F_{k}\right) .
$$

Suppose $\alpha$ is a coefficient in $F_{1}$ not occurring in $\phi$; then it does not appear in $[\phi(a, x)]_{1}$. Since

$$
\phi(a, x)=H_{1} F_{1}+\cdots+H_{k} F_{k}, \ddagger
$$

we have

$$
[\phi(a, x)]_{1}=\left[H_{1}\right]_{1} F_{1} .
$$

* This is the Kronecker substitution.

$\dagger$ König, p. 262.

$\ddagger$ This relation is of course understood to be an identity in the variables $x$ and the coefficients $a$. In particular, $\psi(a)=0$ shall indicate that $\psi$ vanishes identically in the coefficients $a$. 
But $\left[H_{1}\right]_{1}=0$, as otherwise $[\phi(a, x)]_{1}$ and consequently $\phi(a, x)$ would contain $\alpha$. Hence $[\phi(a, x)]_{1}=0$ and from relation (5) (with $m$ replaced by $k$ ) we have the desired result.

Theorem 2.* If

$$
\psi(a) \equiv 0 \quad\left(\bmod F_{1}, F_{2}, \cdots, F_{k}\right)
$$

where $k \leqq m-1$, then $\psi(a)=0$.

It is clearly sufficient to prove the theorem for $k=m-1=\sum_{j=1}^{r} \alpha_{j}$, which is the total number of variables occurring in the polynomials $F_{i}$. The theorem is seen to be true in case $m=2$ as in this case we have a single polynomial in a single variable. We accordingly prove the theorem by induction on the total number of variables in our polynomials. We assume the theorem is true for $\mu-1$ general polynomials in $\mu-1$ variables, no matter how the variables are distributed among the various sets.

Let $G_{1}, G_{2}, \cdots, G_{\mu}$ be general polynomials in a total of $\mu$ variables, and let $\psi$ denote any polynomial in the coefficients of these polynomials satisfying the relation

$$
\psi \equiv 0 \quad\left(\bmod G_{1}, G_{2}, \cdots, G_{\mu}\right) .
$$

By Theorem $1, \psi$ actually contains the coefficient, say $\beta$, of the term $x_{11}^{b}(b>0)$ in $G_{1}$ or

$$
\psi \equiv 0 \quad\left(\bmod G_{2}, G_{3}, \cdots, G_{\mu}\right) .
$$

In the latter case we have the identity,

$$
\begin{aligned}
\psi & =K_{2} G_{2}+\cdots+K_{\mu} G_{\mu} \\
& =\left(K_{2}\right)_{x_{11}=0}\left(G_{2}\right)_{x_{11}=0}+\cdots+\left(K_{\mu}\right)_{x_{11}=0}\left(G_{\mu}\right)_{x_{11}=0} .
\end{aligned}
$$

But $\left(G_{2}\right)_{x_{11}=0}, \cdots,\left(G_{\mu}\right)_{x_{11}=0}$ is a set of $\mu-1$ general polynomials in $\mu-1$ variables, and by the hypothesis of the induction, $\psi=0$. Suppose however that $\psi$ contains $\beta$, and when arranged according to powers of $\beta$ let $\psi_{s}$ be the coefficient of $\beta^{s}(s>0)$, the highest power of $\beta$ occurring in $\psi$. In the identity

$$
\psi=\sum_{i=1}^{\mu} H_{i} G_{i}=H_{1}\left(\cdots+\beta x_{11}^{b}+\cdots\right)+\sum_{i=2}^{\mu} H_{i} G_{i}
$$

equate the coefficients of $\beta^{s}$ on both sides. We get

$$
\psi_{s}=L_{1} x_{11}^{b}+L_{2} G_{2}+\cdots+L_{\mu} G_{\mu},
$$

where $L_{1}=0$ if $H_{1}$ is of degree less than $s-1$ in $\beta$. Place $x_{11}=0$ and we have

* Cf. König, p. 263. 


$$
\psi_{s}=\left(L_{2}\right)_{x_{11}=0}\left(G_{2}\right)_{x_{11}=0}+\cdots+\left(L_{\mu}\right)_{x_{11}=0}\left(G_{\mu}\right)_{x_{11}=0}
$$

By the argument above we find that $\psi_{s}=0$, which contradicts our assumption that $\beta$ actually appeared in $\psi$. Hence $\psi=0$.

2. The fundamental theorem. We have just shown that there exists no polynomial in the coefficients of the polynomials (4) which belongs to the module defined by $F_{1}, F_{2}, \cdots, F_{k}$ where $k \leqq(m-1)$. That there does exist such a polynomial if $k=m$ is shown by Theorem 3 below. Before stating this theorem we need to give a definition.

By the weight of a coefficient of $F_{i}$ with regard to the variables of the kth set, we shall mean the exponent of $x_{k, \alpha k+1}$ in the corresponding term of the homogeneous form $F_{i}^{\prime}$.

For convenience let us set

$$
L_{i}=n_{i 1} t_{1}+n_{i 2} t_{2}+\cdots+n_{i r} t_{r} \quad(i=1,2, \cdots, m)
$$

where the $t$ 's are a set of independent parameters and $n_{i j}$ represents the degree of $F_{i}$ in the variables $x_{j 1}, x_{j 2}, \cdots, x_{j, \alpha_{j}}$, of the $j$ th set. We may now state the following fundamental theorem.

THEOREM 3.* There exists one and only one $\dagger$ rational and integral function, say $R(a)$, of the coefficients of the general polynomials (4) with the following properties:

(a) $R(a)$ is irreducible;

(b) $R(a) \equiv 0\left(\bmod F_{1}, F_{2}, \cdots, F_{m}\right)$;

(c) $R(a)$ is homogeneous and of degree $N_{i}$ in the coefficients of $F_{i}$ separately, where

$$
N_{i}=\text { coefficient of } t_{1}^{\alpha_{1}} t_{2}^{\alpha_{2}} \cdots t_{r}^{\alpha_{r}} \text { in } \prod_{l=1}^{m}{ }^{(i)} L_{l} \ddagger \quad(i=1,2, \cdots, m) \text {; }
$$

(d) $R(a)$ is isobaric of weight $W_{k}$ with regard to the variables of the kth set, where

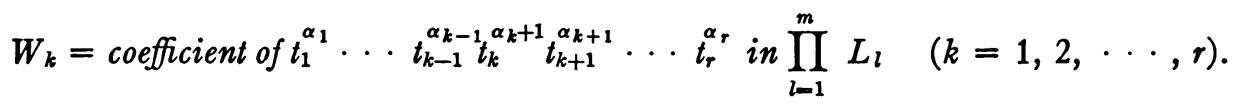

This polynomial $R(a)$ is defined to be the resultant of the polynomials (4), and is thus defined only to within a numerical factor. Lemma 1 below

* Cf. König, p. 271. Parts (c) and (d) of this theorem were stated by Sylvester, Proceedings of the Royal Society of London, vol. 12 (1862-63), pp. 674-76, or Mathematical Papers, vol. 2, pp. 329-330.

$\dagger$ That is, if $R(a)$ and $R^{\prime}(a)$ are two polynomials satisfying these conditions, then they differ by only a numerical factor.

$\ddagger$ This notation indicates, as usual, that $l$ is not to take the value $i$ in this product. 
shows that the resultant is determined by the properties (a) and (b) and accordingly the remaining properties must be consequences of these.

The theorem is known to be true in the ordinary case of one set of variables. We have in this case $r=1, m=1+\alpha_{1}, N_{i}=\left(n_{11} n_{21} \cdots n_{m 1}\right) / n_{i 1}$, $W_{1}=n_{11} n_{21} \cdots n_{m 1}$. However we shall in the proof of the theorem only make use of this fact for the case of two ordinary polynomials in a single variable, which is the case for $m=2$. We now assume the theorem for $m$ general polynomials (4) where $r$ and $\alpha_{j}(j=1,2, \cdots, r)$ are any positive integers such that $m=1+\sum_{j=1}^{r} \alpha_{j}$. We shall show that it holds for $m+1$ general polynomials in a total of $m$ variables.

Let

$$
G_{i} \quad(i=1,2, \cdots, m+1)
$$

be a set of general non-homogeneous polynomials in $s$ sets of variables with $\beta_{j}$ variables in the $j$ th set and $m=\sum_{j=1}^{s} \beta_{j}$. We may without confusion denote the variables of the $j$ th set by $x_{j 1}, x_{j 2}, \cdots, x_{j, \beta_{j}}$. Let $\nu_{i j}(>0)$ be the degree of $G_{i}$ in the variables of the $j$ th set $(i=1,2, \cdots, m+1 ; j=1,2, \cdots, s)$. Further let

$$
\begin{array}{rlrl}
\bar{L}_{i}=\nu_{i 1} t_{1}+\nu_{i 2} t_{2}+\cdots+\nu_{i s} t_{s} & & (i=1,2, \cdots, m+1), \\
\bar{N}_{i}=\text { coefficient of } t_{1}^{\beta_{1}} t_{2}^{\beta_{2}} \cdots t_{s}^{\beta_{s}} \text { in } \prod_{l=1}^{m+1}{ }^{(i)} \bar{L}_{l} & (i=1,2, \cdots, m+1), \\
\bar{W}_{k}=\text { coefficient of } t_{1}^{\beta_{1}} \cdots t_{k-1}^{\beta_{k-1}} t_{k}^{\beta_{k+1}} t_{k+1}^{\beta_{k+1}} \cdots t_{s} \text { in } & \prod_{l=1}^{m+1} \bar{L}_{l}(k=1,2, \cdots, s) .
\end{array}
$$

We wish to show under the hypothesis of the induction that the resultant of the polynomials (6) exists and is of degree $\bar{N}_{i}$ in the coefficients of $G_{i}$ and of weight $\bar{W}_{k}$ with regard to the variables of the $k$ th set.

Before proceeding further we need three lemmas, the first two of which we shall state without proof as they may be readily established as in the case of one set of variables.

Lемма 1. (König, pp. 267, 272-74.) If there exists a polynomial $\phi$ in the coefficients of the general polynomials (6) such that

$$
\phi \equiv 0 \quad\left(\bmod G_{1}, G_{2}, \cdots, G_{m+1}\right),
$$

then there exists one and only one irreducible polynomial $R^{\prime}$ with the same property and $\phi$ is divisible by $R^{\prime}$. Also $R^{\prime}$ is homogeneous in the coefficients of each polynomial separately and isobaric with regard to each set of variables.

The proof of this lemma does not depend upon the hypothesis of the induction. 
LEMMA 2. (König, pp. 274-75.) Let $g(a, x)$ be any polynomial in the coefficients of $F_{1}, F_{2}, \cdots, F_{m}$ and in the variables, of total degree $\lambda$ in the variables. Then

$$
\left(\frac{\partial R(a)}{\partial \gamma_{i}}\right)^{\lambda} g(a, x) \equiv h(a) \quad\left(\bmod F_{1}, F_{2}, \cdots, F_{m}\right),
$$

where $h(a)$ is a polynomial in the coefficients only and $\gamma_{i}$ is the constant term in $F_{i}$. Further, a necessary and sufficient condition that $g(a, x) \equiv 0\left(\bmod F_{1}, F_{2}\right.$, $\left.\cdots, F_{m}\right)$ is that $h(a) \equiv 0\left(\bmod F_{1}, F_{2}, \cdots, F_{m}\right)$.

Lemma 3. ${ }^{*}$ Let $G_{i}(i=1,2, \cdots, m+1)$ be the general polynomials (6) and indicate by $\alpha$ the coefficient of the term $x_{11}^{\nu_{11}} x_{21}^{\nu_{13}} \cdots x_{s 1}^{\nu_{1 s}}$ in $G_{1}$. If $\psi(\neq 0)$ is a polynomial in the coefficients of these polynomials and

$$
\psi \equiv 0 \quad\left(\bmod G_{1}, G_{2}, \cdots, G_{m+1}\right),
$$

then in the development of $\psi$ according to powers of $\alpha$,

$$
\psi_{\mu}=\psi_{0}+\cdots+\psi_{\mu} \alpha^{\mu}
$$

$\psi_{\mu}$ is divisible by $\bar{R}_{1}^{\nu_{11}} \bar{R}_{2}^{\nu_{12}} \ldots \bar{R}_{s}^{\nu_{13}}$, where $\bar{R}_{k}$ is the resultant of the general polynomials, $\left(G_{2}\right)_{x_{k 1}=0}, \cdots,\left(G_{m+1}\right)_{x_{k 1}=0}(k=1,2, \cdots, s)$.

Each $\bar{R}_{k}$ has the properties of Theorem 3 by the hypothesis of the induction, as it is the resultant of a set of $m$ general polynomials in a total of $m-1$ variables.

We have

$$
\psi=H_{1} G_{1}+H_{2} G_{2}+\cdots+H_{m+1} G_{m+1}
$$

and by equating coefficients of $\alpha^{\mu}$ on both sides $\uparrow$ we get

$$
\psi_{\mu}=K_{1} x_{11}^{\nu_{11}} x_{21}^{\nu_{12}} \cdots x_{s 1}^{\nu_{1 s}}+K_{2} G_{2}+\cdots+K_{m+1} G_{m+1} \text {. }
$$

Place $x_{k 1}=0$ and we have

$$
\psi_{\mu}=\left(K_{2}\right)_{x_{k 1}=0}\left(G_{2}\right)_{x_{k 1}=0}+\cdots+\left(K_{m+1}\right)_{x_{k 1}=0}\left(G_{m+1}\right)_{x_{k 1}=0}
$$

and by Lemma $1, \psi_{\mu}$ is divisible by $\bar{R}_{k}(k=1,2, \cdots, s)$.

Suppose $\psi_{\mu}$ is divisible by $\bar{R}_{k}^{\nu_{1 k}-l}$ but not by $\bar{R}_{k}^{\nu_{1 k}-l+1}$. Then we may write

$$
\psi_{\mu}=\bar{R}_{k}^{\nu_{1 k}-l} \eta,
$$

where $\eta$ does not contain $\bar{R}_{k}$ as a factor. From (7) we get

$$
\left[\bar{R}_{k}\right]_{1}^{\nu_{1 k-l}}[\eta]_{1}=K x_{11}^{\nu_{11}} x_{21}^{\nu_{12}} \cdots x_{s 1}^{\nu_{11}}
$$

* This lemma is a generalization of a theorem of Mertens. See König, p. 282. The lemma is stated in unsymmetrical form for convenience of notation.

$\dagger$ The coefficient $\alpha$ actually occurs in $\psi$ by Theorems 1 and 2 , hence $\psi_{\mu} \neq 0$. 
It may now be shown that $\left[\bar{R}_{k}\right]_{1}$ is divisible by $x_{k 1}$ but not by $x_{k 1}{ }^{2}{ }^{*}$ Hence $[\bar{R}]_{1}^{\nu_{1 k}-l}$ is exactly divisible by $x_{k 1}^{\nu_{11 k}-l}$ and thus by (9), $[\eta]_{1}$ is divisible by $x_{k 1}^{l}$. Hence by relation (5) and Lemma $1, \eta$ is divisible by $\bar{R}_{k}$ or $l=0$. But $\eta$ is not divisible by $\bar{R}_{k}$, thus $l=0$ and $\psi_{\mu}$ is divisible by $\bar{R}_{k}^{\nu_{1 k}}$. As each of the resultants $\bar{R}_{1}, \bar{R}_{2}, \cdots, \bar{R}_{s}$ contains coefficients not in any of the others and each is irreducible, it follows that $\psi_{\mu}$ is divisible by the required factor.

Let us assume for the moment the existence of the resultant of the general polynomials (6). We can then use the resultant for the $\psi$ of this lemma. Thus the degree of the resultant in the coefficients of $G_{i}(i=2,3, \cdots, m+1)$ can not be less than the degree to which these coefficients enter the product $\bar{R}_{1}^{\nu_{11}} \bar{R}_{2}^{\nu_{12}} \cdots \bar{R}_{s}^{\nu_{18}}$. That is, the degree of the resultant in the coefficients of $G_{i}$ can not be less than

$$
\begin{aligned}
& \sum_{j=1}^{s} \nu_{1 j}\left(\text { coefficient of } t_{1}^{\beta_{1}} \cdots t_{j-1}^{\beta_{i-1}} t_{j}^{\beta_{j-1}} t_{j+1}^{\beta_{i+1}} \cdots t_{s}^{\beta_{s}} \text { in } \prod_{l=2}^{m+1}{ }^{(i)} \bar{L}_{l}\right) \\
& \quad=\text { coefficient of } t_{1}^{\beta_{1} t_{2}{ }_{2}} \cdots t_{s}^{\beta_{s}} \text { in } \prod_{l=1}^{m+1}{ }^{(i)} \bar{L}_{l}=\bar{N}_{i} \quad(i=2,3, \cdots, m+1) .
\end{aligned}
$$

The polynomial $G_{1}$ played an exceptional part in the statement of Lemma 3 but it is clear that any other one could be used in place of $G_{1}$. By an argument similar to the above we find that the resultant is of degree not less than $\bar{N}_{i}$ in the coefficients of $G_{i}(i=1,2, \cdots, m+1)$. We proceed to show the existence of the resultant and to show that its degree in the coefficients of $G_{i}$ is not greater than $\bar{N}_{i}$.

3. Existence of the resultant. It will be convenient to consider two cases according as $\beta_{j}>1$ for some $j$ or all $\beta_{j}=1$. In the first case we may assume that $\beta_{1}>1$.

Case 1. $\beta_{1}>1$. Let us write the polynomials (6) in the form

$$
G_{i}=\sum A_{\lambda}^{(i)} x_{11}^{\lambda_{1} x_{12}} \cdots x_{1 \beta_{1}}^{\lambda \beta_{1}} \ldots \quad(i=1,2, \cdots, m+1),
$$

where the degrees of the variables of the first set are explicitly indicated in each term. For brevity let $A$ indicate the coefficients in this set of polynomials. These polynomials may also be written in the form

$$
G_{i}=\sum B_{\lambda}^{(i)} x_{11}^{\lambda_{1} x_{12}} \ldots x_{1, \beta_{1-1}}^{\lambda \beta_{1}-1} \cdots(i=1,2, \cdots, m+1)
$$

where $B_{\lambda}^{(i)}$ is a polynomial in $x_{1 \beta_{1}}$ of degree $\nu_{i 1}-\sum_{k=1}^{\beta_{1}-1} \lambda_{k}$. When written in this form we think of the polynomials as polynomials in $s$ sets of variables, there being $\beta_{1}-1$ in the first set and $\beta_{j}$ in the $j$ th set $(j=2,3, \cdots, s)$. Let $B$ denote the aggregate of coefficients $B_{\lambda}^{(i)}$ in this set.

* See König, p. 284. 
The resultant of $G_{1}, G_{2}, \cdots, G_{m}$ when written in the form (11) exists by the hypothesis of the induction. Let us denote it by $R_{m+1}(B)$ or $R_{m+1}\left(A, x_{1 \beta_{1}}\right)$ whenever we wish to show that it depends on the coefficients $A$ and also on $x_{1 \beta_{1}}$. It is seen that $R_{m+1}(A, 0)$ is the resultant of the general polynomials

$$
\left(G_{1}\right)_{x_{1} \beta_{1}=0}, \cdots,\left(G_{2}\right)_{x_{1} \beta_{1}=0} .
$$

Let us calculate the degree of $R_{m+1}\left(A, x_{1 \beta_{1}}\right)$ in $x_{1 \beta_{1}}$. The coefficient $B_{\lambda}{ }^{(i)}$ is of degree $\nu_{i 1}-\sum_{k=1}^{\beta_{1}-1} \lambda_{k}$ in $x_{1 \beta_{1}}$, which is exactly the weight of $B_{\lambda}{ }^{(i)}$ with regard to the $\beta_{1}-1$ variables of the first set. Since this is true for each coefficient and $R_{m+1}(B)$ is isobaric, it is seen by applying Theorem 3 that $x_{1 \beta_{1}}$ occurs in each term of $R_{m+1}\left(A, x_{1 \beta_{1}}\right)$ to the degree $\bar{N}_{m+1}$. The coefficient of this highest power of $x_{1 \beta_{1}}$ in $R_{m+1}\left(A, x_{1 \beta_{1}}\right)$ is not zero, as it is the resultant of the general polynomials obtained from $G_{1}, G_{2}, \cdots, G_{m}$, by replacing each $B_{\lambda}{ }^{(i)}$ by the coefficient of the highest power of $x_{1 \beta_{1}}$ occurring in $B_{\lambda}{ }^{(i)}$.

We know that

$$
R_{m+1}\left(A, x_{1 \beta_{1}}\right) \equiv 0 \quad\left(\bmod G_{1}, G_{2}, \cdots, G_{m}\right) .
$$

Let $b_{i}$ be the constant term in $G_{i}$ when written in the form (11), that is, $b_{i}$ is a polynomial in $x_{1 \beta_{1}}$ but contains no other variables. Denote $\sum_{k=1}^{s} \nu_{m+1, k}$ by $p$. Then by Lemma 2 , we have

$$
\left(\frac{\partial R_{m+1}(B)}{\partial b_{i}}\right)^{p} G_{m+1} \equiv h(B) \quad\left(\bmod G_{1}, G_{2}, \cdots, G_{m}\right) .
$$

We may also write $h(B)$ as $h\left(A, x_{1 \beta_{1}}\right)$. Now $h(B)$ is not zero and is of the first degree in the coefficients of $G_{m+1}$, as $R_{m+1}(B)$ does not contain these coefficients. Suppose $h\left(A, x_{1 \beta_{1}}\right)$ does not actually contain $x_{1 \beta_{1}}$. Then we have

$$
h(A, 0) \equiv 0 \quad\left(\bmod G_{1}, G_{2}, \cdots, G_{m+1}\right),
$$

and by Lemma 1, the resultant of our forms (10) exists and is of at most the first degree in the coefficients of $G_{m+1}$. But we have shown above that the resultant can not be of degree less than $\bar{N}_{m+1}$ in these coefficients and $\bar{N}_{m+1} \geqq 1$. Hence $\bar{N}_{m+1}$ must in this case be equal to 1 and the resultant is of degree $\bar{N}_{m+1}$ in the coefficients of $G_{m+1}$.

Suppose then that $h\left(A, x_{1 \beta_{1}}\right)$ actually contains $x_{1 \beta_{1}}$. It may now be shown that $R_{m+1}\left(A, x_{1 \beta_{1}}\right)$ and $h\left(A, x_{1 \beta_{1}}\right)$ have no common factor other than a numerical constant.* Let $S_{m+1}(A)$ be the ordinary resultant of these two as polynomials in $x_{1 \beta_{1}}$. Then $S_{m+1}(A) \neq 0$ and

or by (12) and (13),

$$
S_{m+1}(A) \equiv 0 \quad\left(\bmod R_{m+1}(B), h(B)\right),
$$

* See König, p. 281. 


$$
S_{m+1}(A) \equiv 0 \quad\left(\bmod G_{1}, G_{2}, \cdots, G_{m+1}\right) .
$$

Lemma 1 again establishes the existence of the resultant of the polynomials (10) and we know that $S_{m+1}(A)$ is divisible by this resultant. Since $R_{m+1}(B)$ is of degree $\bar{N}_{m+1}$ in $x_{1 \beta_{1}}$ and $h\left(A, x_{1 \beta_{1}}\right)$ is linear in the coefficients of $G_{m+1}, S_{m+1}(A)$ is of degree $\bar{N}_{m+1}$ in the coefficients of $G_{m+1}$. Thus the degree of the resultant in the coefficients of $G_{m+1}$ is not greater than $\bar{N}_{m+1}$, and by the result of $\$ 2$ this degree is not less than $\bar{N}_{m+1}$. Hence this degree is exactly $\bar{N}_{m+1}$ as we wished to show.

By a similar argument it may be shown that the degree of the resultant in the coefficients of $G_{i}$ is exactly $\vec{N}_{i}$ for each $i$.

Case 2. $\beta_{j}=1(j=1,2, \cdots, s), m=s$. There is only one variable in each set; let us denote them by $y_{1}, y_{2}, \cdots, y_{\text {s }}$ respectively. The polynomials may be written in the two forms

$$
\begin{aligned}
& G_{i}=\sum A_{\lambda}^{(i)} y_{1}^{\lambda_{1}} y_{2}^{\lambda_{2}} \cdots y_{8}^{\lambda_{8}} \\
& =\sum B_{\lambda}^{(i)} y_{2}^{\lambda_{2}} y_{3}^{\lambda_{2}} \cdots y_{8}^{\lambda_{8}} \quad(i=1,2, \cdots, m+1),
\end{aligned}
$$

where $B_{\lambda}{ }^{(i)}$ is of degree $\nu_{i 1}$ in $y_{1}$.

Let $M_{i}$ denote the coefficient of $t_{2} t_{3} \cdots t_{8}$ in

$$
\prod_{j=1}^{m}(i)\left(\nu_{j 2} t_{2}+v_{j 3} t_{3}+\cdots+\nu_{j s} t_{s}\right) .
$$

Now form the resultant of $G_{1}, G_{2}, \cdots, G_{m}$ as polynomials in $y_{2}, y_{3}, \cdots, y_{s}$, say $R_{m+1}^{\prime}(B)=R_{m+1}^{\prime}\left(A, y_{1}\right)$. Then by the hypothesis of the induction, $R_{m+1}^{\prime}\left(A, y_{1}\right)$ is of degree

$$
M=\nu_{11} M_{1}+\nu_{21} M_{2}+\cdots+\nu_{m 1} M_{m}
$$

in $y_{1}$. But $M$ is the coefficient of $t_{1} t_{2} \cdots t_{s}$ in

$$
\prod_{j=1}^{m}\left(\nu_{j 1} t_{1}+\nu_{j 2} t_{2}+\cdots+\nu_{j s} t_{s}\right)
$$

and thus $M=\bar{N}_{m+1}$. From this point we may proceed exactly as in the previous case and the details will be omitted.

Before considering the proof of part (d) of Theorem 3 it is desirable to pass back to homogeneous forms.

4. The resultant of homogeneous forms. Let us make our general polynomials (6) homogeneous in each of the $s$ sets of variables by introducing new variables, $z_{j}=x_{j, \beta_{j}+1}(j=1,2, \cdots, s)$. These homogeneous forms will be denoted by

$$
G_{i}^{\prime} \quad(i=1,2, \cdots, m+1) .
$$


Let $R$ indicate now the resultant of the polynomials (6). Then we have

$$
R=\sum_{i=1}^{m+1} H_{i} G_{i} .
$$

This goes over in the homogeneous case to

$$
R z_{1}^{\rho_{1}} z_{2}^{\rho_{2}} \cdots z_{s}^{\rho_{s}}=\sum_{i=1}^{m+1} H_{i}^{\prime} G_{i}^{\prime}
$$

where $\rho_{k}$ is the weight of $R$ with regard to the variables of the $k$ th set. We show below that $\rho_{k}=\bar{W}_{k}$ but for the present its value is immaterial.

In $G_{i}^{\prime}$ let us place $x_{1 j_{1}}=x_{2 j_{2}}=\cdots=x_{s, i_{s}}=1$, where these are any variables of the respective sets, and denote the resulting non-homogeneous polynomials by $G_{i}{ }^{(j)}$. Then from (15) we have

$$
R z_{1}^{\rho_{1}} z_{2}^{\rho_{2}} \cdots z_{s}^{\rho_{d}}=\sum_{i=1}^{m+1} H_{i}^{(j)} G_{i}^{(j)}
$$

By the Kronecker substitution we see that $[R]=0$ and thus

$$
R \equiv 0 \quad\left(\bmod G_{1}^{(j)}, G_{2}^{(j)}, \cdots, G_{m+1}^{(j)}\right) .
$$

If we denote by $R^{(j)}$ the resultant of the polynomials $G_{i}{ }^{(j)}$, then this relation shows that $R$ is divisible by $R^{(i)}$ and by reversing the process we see that $R^{(j)}$ is divisible by $R$. Thus we get the same resultant, defined only to within a numerical factor, no matter which one of the variables of each set in $G_{i}$ we place equal to unity. The resultant $R$ of $G_{i}(i=1,2, \cdots, m+1)$ we accordingly define to be the resultant of the homogeneous forms (14).

Let $\bar{G}_{i}^{\prime}(i=1,2, \cdots, m+1)$ denote the set of forms (14) after we have made a general non-singular linear transformation on the variables of say the first set, and let $\bar{R}$ be the resultant of this transformed set of forms. It follows immediately* that

$$
\bar{R}=R U,
$$

where $U$ is a form in the coefficients of the transformation only. As a matter of fact $U$ must be a power of the determinant of the transformation. $\dagger$ As a special case we see that the resultant is unchanged if the variables are permuted in any way within the set in which they occur.

We now prove the following:

* König, p. 293.

† See Bôcher, Higher Algebra, p. 220. Thus the resultant is an invariant under independent linear transformations of the various sets. 
LemMa 4. Let $x_{1 j_{1}}, x_{2 j_{2}}, \cdots, x_{s j_{s}}$ be any variables of the respective sets in $G_{i}^{\prime}$. Then the resultant of the forms $G_{i}^{\prime}$ contains the coefficient of

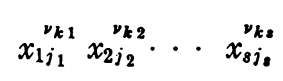

in $G_{k}^{\prime}$ to the degree $\bar{N}_{k}$.

In view of the previous remarks it will be sufficient to prove this lemma for the case where $j_{r}=\beta_{r}+1(r=1,2, \cdots, s)$. For convenience of notation let us consider the case $k=2$. Let $\alpha_{2}$ denote the coefficient under consideration, that is, $\alpha_{2}$ is the constant term in $G_{2}$. By Lemma 3, $\alpha_{2}$ enters the resultant to a degree as great as the degree to which it enters $\bar{R}_{1}^{\nu_{11}} \bar{R}_{s}^{\nu_{12}} \cdots \bar{R}_{s}^{\nu_{10}}$, which by the use of induction on the number of variables is

$$
\sum_{k=1}^{\delta} \nu_{1 k}\left[\text { coefficient of } t_{1}^{\beta_{1}} \cdots t_{k-1}^{\beta_{k-1}} t_{k}^{\beta_{k-1}} t_{k+1}^{\beta_{k+1}} \cdots t_{s}^{\beta_{s}} \text { in } \prod_{i=3}^{m+1}\left(\nu_{i 1} t_{1}+\cdots+\nu_{i s} t_{s}\right)\right] \text {. }
$$

But this is $\bar{N}_{2}$. The proof is unchanged for any $k \neq 1$. If $k=1$, we need only to change the way in which Lemma 3 has been stated for simplicity of notation.

It now follows from Lemma 3 by a consideration of the degrees that the resultant of the forms (14) contains the term

$$
\alpha^{\bar{N}_{1}} \bar{R}_{1}^{\nu_{11}} \bar{R}_{2}^{\nu_{12}} \cdots \bar{R}_{\delta}^{\nu_{1 \bullet}}
$$

with at most a numerical coefficient. Here $\alpha$ is the coefficient of $x_{11}^{\nu_{11}} x_{21}^{\nu_{12}} \cdots x_{s 1}^{\nu_{18}}$ in $G_{1}$. We may determine the weight of this expression (17) with regard to the $k$ th set of variables by the hypothesis of the induction and it is found to be $\bar{W}_{k}$. Since by Lemma 1 the resultant is isobaric, each term of the resultant is of weight $\bar{W}_{k}$ with regard to the $k$ th set of variables. This proves part (d) of Theorem 3 and thus completes the proof of the theorem.

\section{THE RESUlTANT IN DETERMINANT FORM}

5. We now pass to the problem of expressing the resultant in determinant form in certain special cases.

Let

$$
\phi_{i} \quad(i=1,2, \cdots, m)
$$

be a set of general forms homogeneous in each of $s+t=r(r \geqq 1, s, t \geqq 0)$ sets of variables, there being $\alpha_{j}+1$ variables in the $j$ th set $\left(\alpha_{j} \geqq 1, j=1,2, \cdots, r\right)$. We assume further that $\alpha_{1}=\alpha_{2}=\cdots=\alpha_{s}=1$, and hence $m=s+1+\sum_{s+1}^{r} \alpha_{j}$. Also the degrees of these forms in the various sets are assumed to be those given in the following table: 


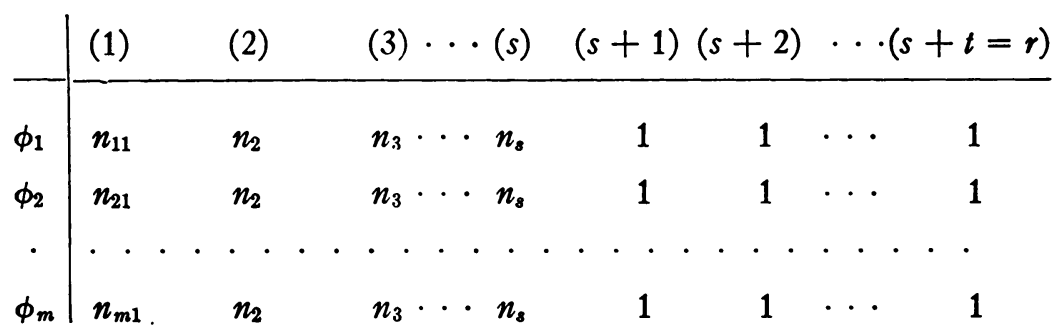

Here the degree of $\phi_{i}$ in the variables of the $j$ th set is found at the intersection of the $i$ th row and $j$ th column. The numbers $n_{j 1}(j=1,2, \cdots, m), n_{2}, n_{3}$, $\cdots, n_{s}$ are arbitrary positive integers.

We shall express in determinant form the resultant of this system of forms. As a special case if $t=0$, we have a set of multiple binary forms, the degree in the variables of the $k$ th set being the same for each form if $k>1$. If, further, $r=1$, we have two ordinary binary forms of arbitrary degrees and our form of the resultant reduces to the Sylvester determinant. If on the other hand $s=0$, we have a set of forms linear in each of $t$ sets of variables, there being an arbitrary number of variables in each set.

We now state the principal result of this section.

Theorem 4. Let $\phi_{i}(i=1,2, \cdots, m)$ be the general forms (18) and consider all possible equations of the type

$$
\rho_{i} \phi_{i}=0 \quad(i=1,2, \cdots, m) .
$$

Here $\rho_{i}$ represents a power product of the variables of such a degree that $\rho_{i} \phi_{i}$ is homogeneous of degree $\sum_{i=1}^{m} n_{i 1}-1$ in the variables of the first set; of degree $(m-k+1) n_{k}-1$ in the variables of the kth set $(k=2,3, \cdots, s)$; of degree $\alpha_{s+l+1}+\cdots+\alpha_{r}+1$ in the variables of the $(s+l)$ th set $(l=1,2, \cdots, t-1)$; and of the first degree in the variables of the rth set.* Considering these power products of the variables as unknowns, we have in the set (20) the same number of equations as unknowns and the determinant of the coefficients of the unknowns is the resultant of the given forms.

Let us calculate, for example, the number of the equations (20) arising from $\phi_{1}$. Making use of the fact that the number of terms in a general polynomial, homogeneous of degree $N$ in one set of $M$ variables, is

$$
\left(\begin{array}{c}
M+N-1 \\
N
\end{array}\right)=\frac{(M+N-1) !}{N !(M-1) !}
$$

we see that the number of equations arising from $\phi_{1}$ is

* That is, $\rho_{i}$ does not contain the variables of the $r$ th set if $t>0$. 


$$
\begin{gathered}
\left(\sum_{i=2}^{m} n_{i 1}\right)(m-2)(m-3) \cdots(m-s) n_{2} n_{3} \cdots n_{s}\left(\begin{array}{c}
\alpha_{s+1}+\alpha_{s+2} \cdots+\alpha_{r} \\
\alpha_{s+1}
\end{array}\right) \\
\times\left(\begin{array}{c}
\alpha_{s+2}+\cdots+\alpha_{r} \\
\alpha_{s+2}
\end{array}\right) \cdots\left(\begin{array}{c}
\alpha_{r-1}+\alpha_{r} \\
\alpha_{r-1}
\end{array}\right) .
\end{gathered}
$$

Remembering that $m=s+1+\sum_{s+1}^{r} \alpha_{j}$, this may be reduced to the form

$$
\left(\sum_{i=2}^{m} n_{i 1}\right) n_{2} n_{3} \cdots n_{s}[(m-2) !] /\left(\alpha_{s+1} ! \alpha_{s+2} ! \cdots \alpha_{r} !\right)
$$

The total number of the equations (20) is found to be

$$
\left(\sum_{i=1}^{m} n_{i 1}\right) n_{2} n_{3} \cdots n_{s}[(m-1) !] /\left(\alpha_{s+1} ! \alpha_{s+2} ! \cdots \alpha_{r} !\right)
$$

and a calculation similar to the above shows that this is also the total number of unknowns. We thus have the same number of equations as unknowns.

Let $D$ be the determinant of the coefficients of the unknowns in the equations (20). Assume for the present that $D \neq 0$ for general forms (18) which we are considering. Let $\beta_{1}, \beta_{2}, \cdots, \beta_{r}$ denote the degrees of the power products in the several sets of variables in equations (20) and suppose that the elements of the last column of $D$ are the coefficients of

$$
x_{12}^{\beta_{1}} x_{22}^{\beta_{2}} \cdots x_{s 2}^{\beta_{2}} \cdots x_{r, \alpha_{r}+1}^{\beta_{r}}
$$

in these equations. Multiply each column of $D$ by the power product of which its elements are coefficients and add to the last column. Each element of the last column is now of the form $\rho_{i} \phi_{i}$. Hence if we expand $D$ in terms of the elements of the last column we get

$$
D x_{12}^{\beta_{1}} x_{22}^{\beta_{2}} \cdots x_{s 2}^{\beta_{s}} \cdots x_{r, \alpha_{r+1}}^{\beta_{r}} \equiv 0 \quad\left(\bmod \phi_{1}, \phi_{2}, \cdots, \phi_{m}\right) .
$$

From the discussion in $\$ 4$ and Lemma 1 it follows that $D$ is divisible by the resultant of the given forms.

Now $D$ is clearly homogeneous of degree given by the expression (21) in the coefficients of $\phi_{1}$. Let us calculate by Theorem 3 the degree of the resultant in these coefficients. This degree is the coefficient of $t_{1} t_{2} \cdots t_{s}$ $\cdot t_{s+1}^{\alpha_{s+1}} \cdots \cdot t_{r}^{\alpha_{r}}$ in

$$
\prod_{i=2}^{m}\left(n_{i 1} t_{1}+n_{2} t_{2}+\cdots+n_{s} t_{s}+t_{s+1}+\cdots+t_{r}\right) .
$$

But this is 


$$
\begin{aligned}
\left(\sum_{i=2}^{m} n_{i 1}\right)\left[\text { coefficient of } t_{2} \cdots t_{s} t_{s+1}^{\alpha_{s+1}} \cdots t_{r}^{\alpha_{r}}\right. \text { in } \\
\left.\quad\left(n_{2} t_{2}+\cdots+n_{s} t_{s}+t_{s+1}+\cdots+t_{r}\right)^{m-2}\right] \\
=\left(\sum_{i=2}^{m} n_{i 1}\right) n_{2} n_{3} \cdots n_{s}[(m-2) !] /\left(\alpha_{s+1} ! \alpha_{s+2} ! \cdots \alpha_{r} !\right) .
\end{aligned}
$$

Thus the degree of $D$ in the coefficients of $\phi_{1}$ is the same as the degree of the resultant in these coefficients and similarly for each $\phi_{i}(i=2,3, \cdots, m)$. As $D$ contains the resultant as a factor, $D$ must be the resultant provided $D \not \equiv 0$.

We proceed to show that $D \not \equiv 0$ by a process of induction. We assume Theorem 4 for the case of the proper number of forms of the general type (18) in fewer variables. It is known to be true for the case of two ordinary binary forms.

Let $\omega_{i}(i=1,2, \cdots)$ represent the power products of the variables occurring in the equations (20), that is, the power products of the degrees mentioned in the statement of Theorem 4 . We first of all specialize $\phi_{1}$ by placing

$$
\phi_{1}=\phi_{1}^{\prime}=x_{11}^{n_{11}} x_{21}^{n_{2}} \cdots x_{s_{1}}^{n_{s}} x_{s+1,1} \cdots x_{r 1} .
$$

Then in each row of $D$ arising from $\phi_{1}^{\prime}$ we have one and only one element different from zero and it is equal to unity. The columns of $D$ in which a 1 thus occurs are those whose elements are coefficients of an $\omega_{i}$ which is divisible by $\phi_{1}^{\prime}$. Let us strike these rows and columns from $D$ and denote the remaining determinant by $D^{\prime}$. Thus $D^{\prime}= \pm D$.

The power products $\omega_{i}$ not divisible by $\phi_{1}^{\prime}$ may be arranged in mutually exclusive sets as follows. Let $\omega_{1}^{(q)}$ denote those which contain $x_{11}$ exactly to the degree $q\left(q=0,1, \cdots, n_{11}-1\right) ; \omega_{p}^{(q)}$ those divisible by $x_{11}^{n_{11}} x_{21}^{n_{2}} \cdots x_{p-1}^{n_{p-1}}$ and containing $x_{p 1}$ to the degree $q\left(q=0,1, \cdots, n_{p}-1 ; p=2,3, \cdots, s\right)$; $\omega_{s+k}$ those divisible by $x_{11}^{n_{11}} x_{21}^{n_{2}} \cdots x_{s 1}^{n_{s}} x_{8+1,1} \cdots x_{8+k-1,1}$ but not divisible by $x_{s+k, 1}(k=1,2, \cdots, r-s)$. Each power product $\omega_{i}$ falls into one and only one of these sets or is divisible by $\phi_{1}^{\prime}$.

Consider now the set of all power products $\rho$ multiplying $\phi_{2}, \cdots, \phi_{m}$, in equations (20). These are of various degrees. The same power product may occur multiplying different forms; in this case we count it as many times as it appears. We suppose further that these $\rho$ 's are so labeled that having given a particular $\rho$ we know which form it multiplies in the equations (20). Thus specifying a given $\rho$ designates a row of $D^{\prime}$. We now define $\rho_{1}^{(q)}, \rho_{p}^{(q)}$ and $\rho_{s+k}$ by the same conditions used in defining $\omega_{1}^{(q)}, \omega_{p}^{(q)}$ and $\omega_{s+k}$ respectively. 
In particular $\rho_{1}^{(q)}$ is the set of $\rho$ 's which contain $x_{11}$ to the degree $q(q=0,1$, $\left.\cdots, n_{11}-1\right)$, and so on. To get this set we first select those multipliers of $\phi_{2}$ with this property, then those multiplying $\phi_{3}$, and so on, each power product being taken as many times as it appears. Each $\rho$ falls into one and only one of the above sets and none of them is divisible by $\phi_{1}^{\prime}$.

A direct calculation shows that the number of elements in the sets $\rho_{1}^{(q)}, \rho_{p}^{(q)}, \rho_{s+k}$ is exactly the number of power products $\omega$ in the sets $\omega_{1}^{(q)}$, $\omega_{p}^{(q)}$ and $\omega_{s+k}$ respectively. By a proper arrangement of rows and columns we may therefore write $\pm D^{\prime}$ in the form

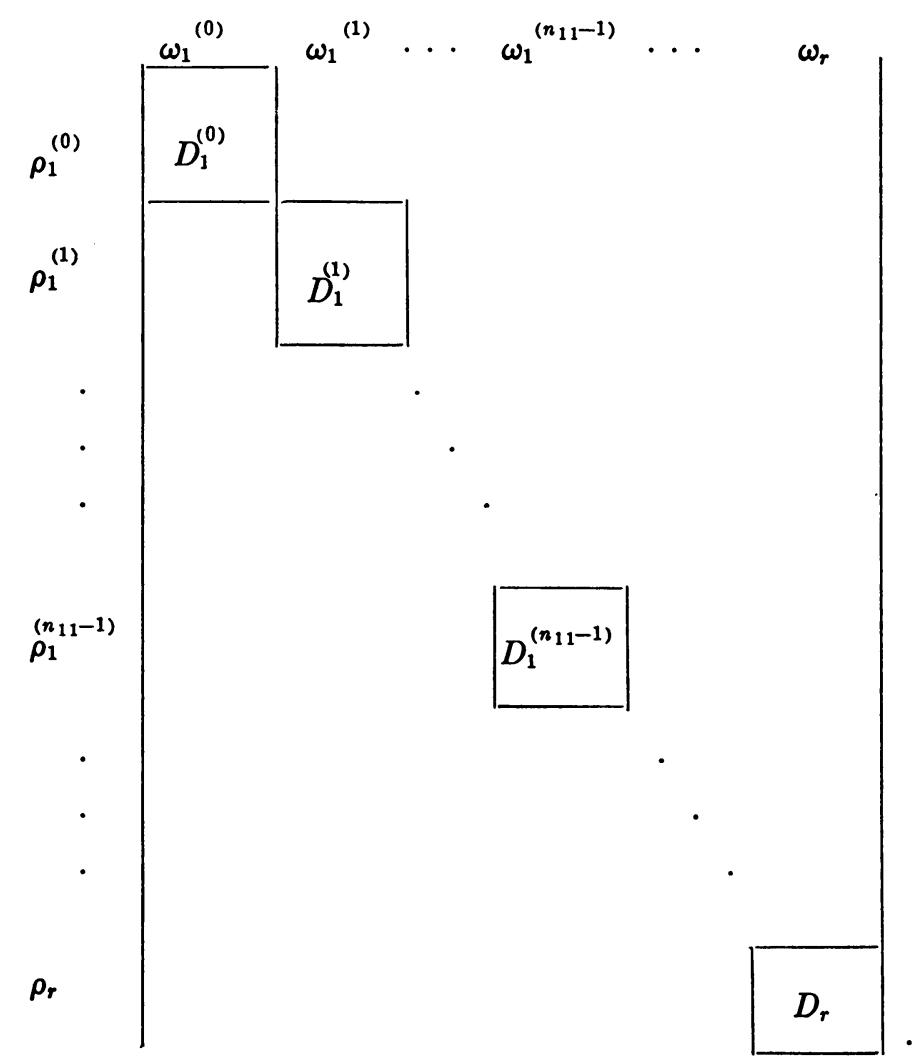

In this arrangement, the elements falling in the square array $D_{1}{ }^{(0)}$ are those in a row of $D^{\prime}$ denoted by a $\rho$ of the set $\rho_{1}{ }^{(0)}$ and in a column designated by an $\omega$ of the set $\omega_{1}{ }^{(0)}$, and so on. We suppose the order of the sets of $\omega$ 's from left to right is

$$
\omega_{1}^{(0)}, \cdots, \omega_{1}^{\left(n_{11}-1\right)}, \omega_{2}^{(0)}, \cdots, \omega_{2}^{\left(n_{2}-1\right)}, \cdots, \omega_{s}^{\left(n_{s}-1\right)}, \omega_{s+1}, \cdots, \omega_{r} .
$$


The $\rho$ 's are arranged in the same order from top to bottom. Now there is no non-zero element vertically below any of the square arrays $D$. For example, consider the set of columns $\omega_{p}^{(q)}(p>1)$. Every set of $\rho$ 's following $\rho_{p}^{(q)}$ contains

$$
x_{11}^{n_{11}} x_{21}^{n_{2}} \cdots x_{p-1,1}^{n_{p}} x_{p 1}^{q+1}
$$

as a factor, while $\omega_{p}^{(q)}$ does not contain this term as a factor. This observation is all that is necessary to obtain this result.

Thus we have

$$
D^{\prime}= \pm D_{1}^{(0)} \cdots D_{1}^{\left(n_{11}-1\right)} \cdots D_{r},
$$

where these denote the determinants of the arrays indicated.

Let $S_{k}$ denote the resultant of

$$
\left(\phi_{2}\right)_{x_{k 1-0}}, \cdots,\left(\phi_{m}\right)_{x_{k 1-0}} \quad(k=1,2, \cdots, r)
$$

with the understanding that if $\alpha_{k}=1$, as is certainly the case for $k=1,2, \cdots, s$, we also place $x_{k 2}=1$. It may now be shown that

$$
\begin{array}{rlrl}
D_{1}^{(q)} & =S_{1} \\
D_{p}^{(q)} & =S_{p} \\
D_{s+k} & =S_{s+k} & (p=2,3, \cdots, s ; q & \left.=0,1, \cdots, n_{p}-1\right), \\
& & (k=1,2, \cdots, r-s) .
\end{array}
$$

We make the calculation for a typical case, say $D_{s}^{(q)}$ for convenience. Denote by $\bar{\phi}_{2}, \cdots, \bar{\phi}_{m}$ the general forms obtained from $\phi_{2}, \cdots, \phi_{m}$ by placing $x_{t 1}=0, x_{s 2}=1$ in each form. Then apply Theorem 4 , as it is true by the hypothesis of the induction. We shall use the notation as above, for example the $r$ th set of variables will denote those variables which belonged to the $r$ th set in the forms (18) although it is only the $(r-1)$ st set here, as the $s$ th set is lacking. We have then equations of the type

$$
\eta_{i} \bar{\phi}_{i}=0 \quad(i=2,3, \cdots, m),
$$

where $\eta_{i} \bar{\phi}_{i}$ is of degree $\sum_{i=2}^{m} n_{i 1}-1$ in the variables of the first set; of degree $(m-k) n_{k}-1$ in the variables of the $k$ th set $(k=2,3, \cdots, s-1)$; of degree $\alpha_{s+l+1}+\cdots+\alpha_{r}+1$ in the variables of the $(s+l)$ th set $(l=1,2, \cdots, t-1)$; and of the first degree in the variables of the $r$ th set. But we obtain exactly these power products occurring in the equations (25) if we divide those of the set $\omega_{s}^{(q)}$ by their common factor,

$$
x_{11}^{n_{11}} x_{21}^{n_{2}} \cdots x_{s-1,1}^{n_{8}-1} x_{81}^{q} x_{s 2}^{n_{8}-q} .
$$


Similarly we obtain all these power products $\eta_{i}$ in (25) by dividing those of the set $\rho_{p}^{(q)}$ by this same factor.

Now the determinant of the coefficients of the unknowns in the equations (25) is $S_{s}$. Multiply these equations by the common factor of the elements of $\omega_{s}^{(q)}$, and we have the equivalent system of equations

$$
\rho_{s}^{(q)} \bar{\phi}_{i}=0 \quad(i=2,3, \cdots, m) .
$$

The determinant of the coefficients in these equations is $D_{s}^{(q)}$ as the power products in this set of equations are exactly those of $\omega^{(q)}$, and $D_{s}^{(q)}$ is seen to contain no coefficient not occurring in the forms $\bar{\phi}_{2}, \cdots, \bar{\phi}_{m}$. Thus we see that $D_{s}^{(q)}=S_{s}$. In a like manner the other relations (24) may be verified. We have then that

$$
D^{\prime}=S_{1}^{n_{11}} S_{2}^{n_{2}} \cdots S_{s}^{n_{s}} S_{s+1} \cdots S_{r}
$$

and no one of these factors is zero. Thus for general forms $D$ is not zero. This completes the proof of Theorem 4 .

The form of the determinant $D$ obtained for a given system of forms (18) clearly depends upon the convention as to which set of variables is the second, which the third, and so on. Thus in general we have a variety of determinantal expressions for the resultant of forms of the type (18).

Surth College, NORTHAMPTON, Mass. 